Esta publicación cientifica en formato digital es continuidad de la revista impresa ISSN-Versión Impresa 0798-1406 / ISSN-Versión on line 2542-3185Depósito legal pp
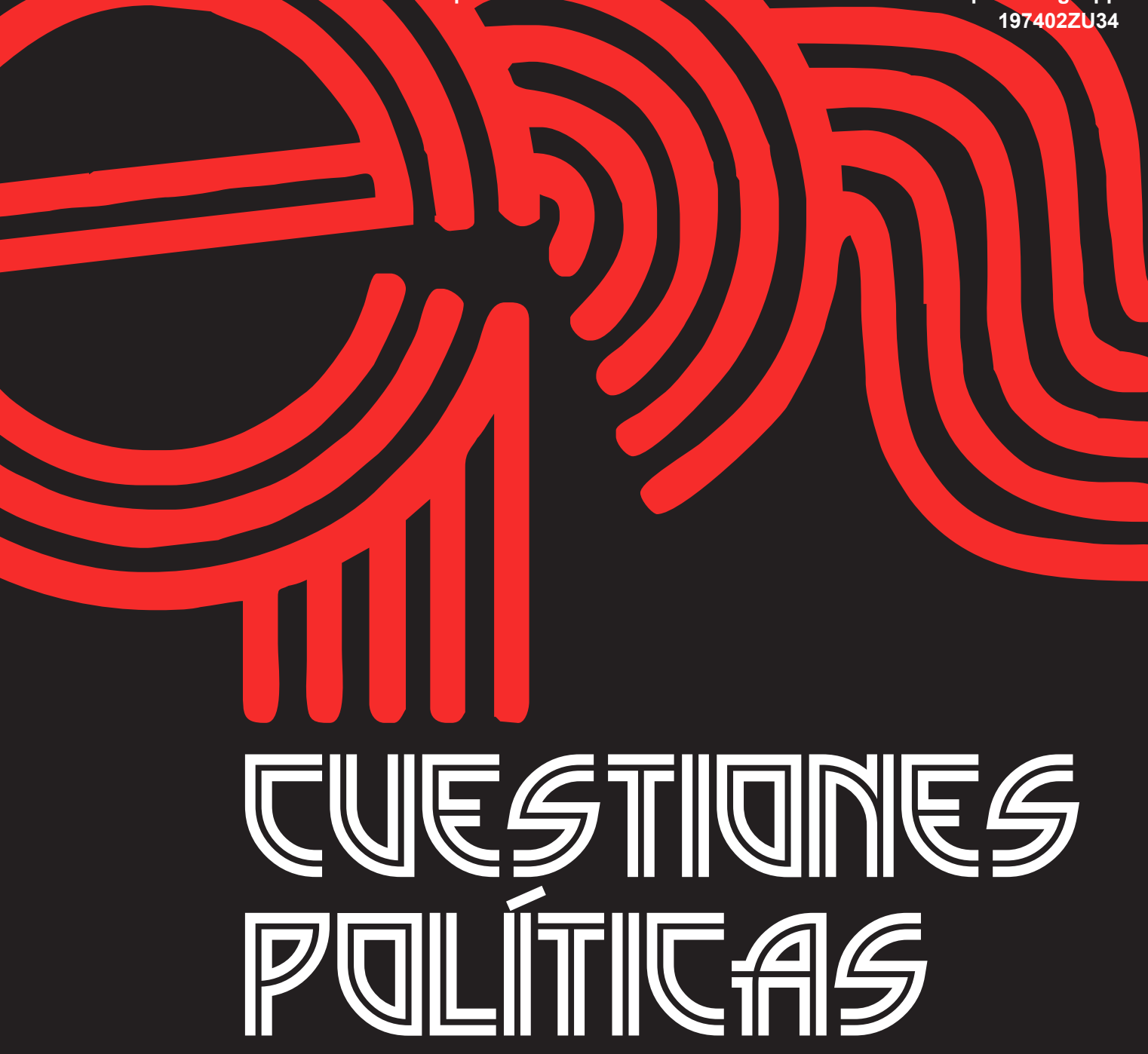

Instituto de Estudios Políticos y Derecho Público "Dr. Humberto J. La Roche" de la Facultad de Ciencias Jurídicas y Políticas de la Universidad del Zulia Maracaibo, Venezuela
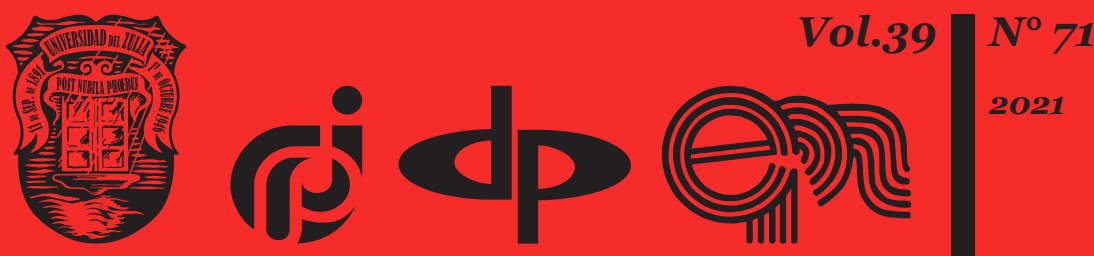


\title{
El Fenómeno del votante ucraniano moderno: esencia, peculiaridades y tendencias de su desarrollo
}

DOI: https://doi.org/10.46398/cuestpol.3971.23

\author{
Roman Oleksenko * \\ Bogdan Malchev ** \\ Olga Venger *** \\ Tetiana Sergiienko **** \\ Olena Gulac *****
}

\section{Resumen}

El artículo revela las peculiaridades del votante ucraniano moderno como un fenómeno especial en la ciencia política. El objetivo principal de la investigación es formar un retrato del votante moderno basado en los datos de algunas encuestas sociológicas, así como abordar la aparición y formación de la imagen de un candidato deseable para el votante. Se utilizaron métodos de análisis histórico, estadísticos, así como el método de comparación. En los resultados destacan que en la personalidad del votante entenderemos a un sujeto que hace una elección consciente de esa figura política que él (el votante) considera capaz de resolver problemas urgentes de la vida, tanto del Estado como de su persona. En este sentido, la atención se centra en revelar los estados de ánimo en la sociedad ucraniana moderna, para describir la imagen de "un candidato ideal" a los ojos de un votante moderno. Se prestó especial atención a la personalidad como elemento integral del espacio sociopolítico y la cosmovisión del ámbito político y electoral. Se concluye que el contexto histórico es muy importante en la formación del entorno político ucraniano, lo que dejar entrever analíticamente las peculiaridades del significado del acto del sufragio.

Palabras Clave: votante ucraniano; cardiocentrismo; cosmovisión política; absentismo; comportamiento electoral.

\footnotetext{
* Dmytro Motornyi Tavria State Agrotechnological University (Melitopol, Ukraine). ORCID ID: https:// orcid.org/oooo-0002-2171-514X

** Dmytro Motornyi Tavria State Agrotechnological University (Melitopol, Ukraine). ORCID ID: https:// orcid.org/o0oo-0002-8002-6395

*** Zaporizhzhia National University, (Zaporizhzhia, Ukraine). ORCID ID: https://orcid.org/oooo-ooo23758-7891

**** National University «Zaporizhzhia Polytechnic», (Zaporizhzhia, Ukraine). ORCID ID: http://orcid. org/oooo-0002-4654-9248

***** National University of Life and Environmental Sciences of Ukraine, (Kyiv, Ukraine). ORCID ID: https://orcid.org/oooo-0001-9004-0185
} 


\title{
The Phenomenon of the Modern Ukrainian Voter: Essence, Peculiarities and Trends of its Development
}

\begin{abstract}
The article reveals the peculiarities of the modern Ukrainian voter as a special phenomenon in political science. The main objective of the research is to form a portrait of the modern voter based on data from some sociological surveys, as well as to address the emergence and formation of the image of a desirable candidate for the voter. Historical and statistical analysis methods as well as the comparison method were used. In the results they emphasize that in the personality of the voter we will understand a subject who makes a conscious choice of that political figure that he (the voter) considers capable of solving urgent problems of life, both State and of his person. In this regard, the focus is on revealing the moods in modern Ukrainian society, to describe the image of "an ideal candidate" in the eyes of a modern voter. Special attention was paid to personality as an integral element of the socio-political space and the worldview of the political and electoral sphere. It is concluded that the historical context is very important in the formation of the Ukrainian political environment, which makes us glimpse analytically the peculiarities of the meaning of the act of suffrage.
\end{abstract}

Keywords: Ukrainian voter; cardiocentrism; political worldview; absenteeism; electoral behavior.

\section{Introducción}

\section{Presentación del material principal}

A principios del siglo XXI, el fenómeno del votante moderno se ha convertido en un elemento fundamental y básico que determina la imagen del sistema político ucraniano y, en consecuencia, crea en su desarrollo dialéctico características especiales de la democracia como el poder sustantivo del pueblo, y penetra en toda la vida social del país.

La participación cada vez más baja en las elecciones, la disminución de la confianza en el sistema político y sus instituciones, la decepción en el Estado como mecanismo de gobernanza efectiva y, otros factores negativos del fenómeno electoral moderno, así como la realidad política y cultural actual indican la confusa conexión de la modernidad con el pasado y el futuro de Ucrania. De ahí que, la importancia de estudiar el fenómeno del votante moderno radica en su fundamentalidad, porque el votante es un indicador y al mismo tiempo un participante en la formación y posterior desarrollo del Estado; un instrumento sutil que permite determinar, en lo 
objetivo y subjetivo, el grado de éxito y corrección del curso de desarrollo de la sociedad y los políticos como parte de un sistema multidimensional, complejo y contradictorio.

Caracterizando el contenido moderno de la política ucraniana, los científicos están tratando de identificar la esencia, las características y los diferentes enfoques del análisis del fenómeno del votante moderno, que se basa en la clave del lugar y el papel de Ucrania como estado independiente, su orientación política y cultural, los procesos de evolución de la sociedad ucraniana. Compartimos las opiniones de Z. Samchuk y enfatizamos que, a lo largo de los siglos, las eras, se reemplazaron entre sí, la autoconciencia ucraniana se formó bajo la influencia de muchos factores: desde la física y geografía socioeconómica, que se formó y esas características políticas y culturales, que crearon el fenómeno del votante moderno.

El fenómeno del votante moderno es una situación sociocultural específica, un fenómeno especial en la ciencia política y la filosofía. Es importante establecer la secuencia, las peculiaridades de la formación de la autoconciencia "humano-votante", las regularidades de su formación y desarrollo, para una comprensión integral de Ucrania como estado independiente y especifica comunidad política y cultural.

En la mente del votante hay un proceso constante de comparar la supuesta meta y el resultado obtenido, la meta y los medios, el ajuste entre la meta, los medios y el resultado. Según Hegel, "El triste deambular del espíritu en uno mismo".

\section{1. "Enfoque histórico" de la evaluación del fenómeno electoral moderno}

Analizando el fenómeno del votante moderno ucraniano se utiliza un "enfoque histórico" que se basa en el complejo cultural, político y social de ideas, valores, creencias que influyen activamente en cada persona con conciencia política. El enfoque histórico es un factor formativo en cualquier fenómeno, ya que la historia es el pasado, y el pasado da forma al presente, tanto como el presente da forma al futuro. La era soviética tuvo un efecto revelador en la formación de la conciencia del votante ucraniano. Sin embargo, esto no significa que la contribución de tiempos pasados no deba subestimarse: desde las primeras tribus eslavas y durante el período imperial, cada época dejó su marca especial en la formación de la autoconciencia, la cosmovisión y la identidad propia del ucraniano contemporáneo. Como ejemplo, podemos mencionar la actividad de Zemsky Sobors (1613) y Zaporizhian Sich. 
La era soviética dio forma a las instituciones socioculturales básicas que operaban en todas las etapas de los procesos electorales, desde el presidente hasta los diputados del pueblo de todas las convocatorias. Su tarea principal era reducir la participación continua y consciente del individuo en el proceso electoral.

La peculiaridad del período de la era soviética era que el votante de la URSS no tenía experiencia de elección política. Al mismo tiempo, los ciudadanos de Europa y de los Estados Unidos tienen siglos de experiencia en la sociedad civil y política. El elector recibió un "producto terminado" por el que tuvo que votar. Así como no había "democracia de vida" - como el derecho a elegir y responder por su elección.

Coincidimos con los puntos de vista de V. Sabadukh, y enfatizamos que la reestructuración cardinal de la conciencia pública, la cultura, la estructura de la sociedad a la que nuestro país fue sometido en esta época puso una marca especial en la formación de una imagen de un votante moderno. Durante los años de existencia de la URSS, la conciencia de generaciones enteras ha sufrido una metamorfosis radical, de generación en generación, en la conciencia de todos fortaleció la nueva realidad - socialista.

La era, cambió para siempre a todos los ucranianos que nacieron en la URSS. Después de su colapso, a pesar de la edad y el género, en mayor o menor medida, se ha creado una imagen completamente nueva de un político, mediante los conceptos de uso generalizado de: "persona políticaeconómica", "hombre interior", "hombre del pueblo". En las realidades de hoy se entiende más bien como un término de ciencia política.

Se formó un estereotipo en la conciencia pública según el cual sólo "un hombre del pueblo" que era capaz de entender al pueblo, para formar parte de él, puede así "dar" al pueblo esperanza y prosperidad, y un sentido efímero de estabilidad. En la autoconciencia del votante ucraniano, el "hombre interior" fue capaz de proporcionar estabilidad en el Estado y en la vida pública.

La imagen de la "persona económica" se basa en el "cardiocentrismo ucraniano", un concepto que se originó en la filosofía ucraniana del el siglo XIX, en las obras de G. Skovoroda, Gogol, P. Yurkevich,T. Shevchenko y P. Kulish (Fidrowska, 2019). El cardiocentrismo es una doctrina idealista en la filosofía ucraniana, cuyo desarrollo político ha tenido un efecto especial en los últimos siglos de la formación de la nación, fortalecida como término politológico. En el sentido politológico, se puede considerar como una doctrina de la ciencia política ucraniana moderna, que determina la forma de pensar de los votantes en el contexto de una imagen idealizada del "hombre del alma" (sobre el "cardiocentrismo" del votante ucraniano). Fueron los filósofos ucranianos quienes presentaron el cardiocentrismo ucraniano como un fenómeno cultural, caracterizado por la mentalidad 
nacional y otros factores socioculturales. Según esta interpretación, el cardiocentrismo es esencialmente una doctrina sobre el alma, sobre su predominio sobre el cuerpo, en su encarnación materialista.

La visión de la imagen de la "persona económica" es una característica diferenciadora del campo electoral ucraniano. Desarrollado por tecnólogos políticos y propagado por todos los medios de comunicación e impuesto al votante mediante la idea de una persona candidata-económica que como resultado lo lleva a la victoria. Como sabemos, la imagen de V. Zelensky como futuro presidente se formó sobre la base de la película «Siervo del Pueblo», donde se creó una imagen positiva para consumo general, que hizo que la campaña electoral fuera exitosa.

\section{La imagen de la "persona económica" en las condiciones de los sentimientos electorales modernos}

En nuestra opinión, la imagen de "persona económica" es, en primer lugar, la encarnación de la competencia y el profesionalismo, la integridad y la firmeza en la toma de decisiones.

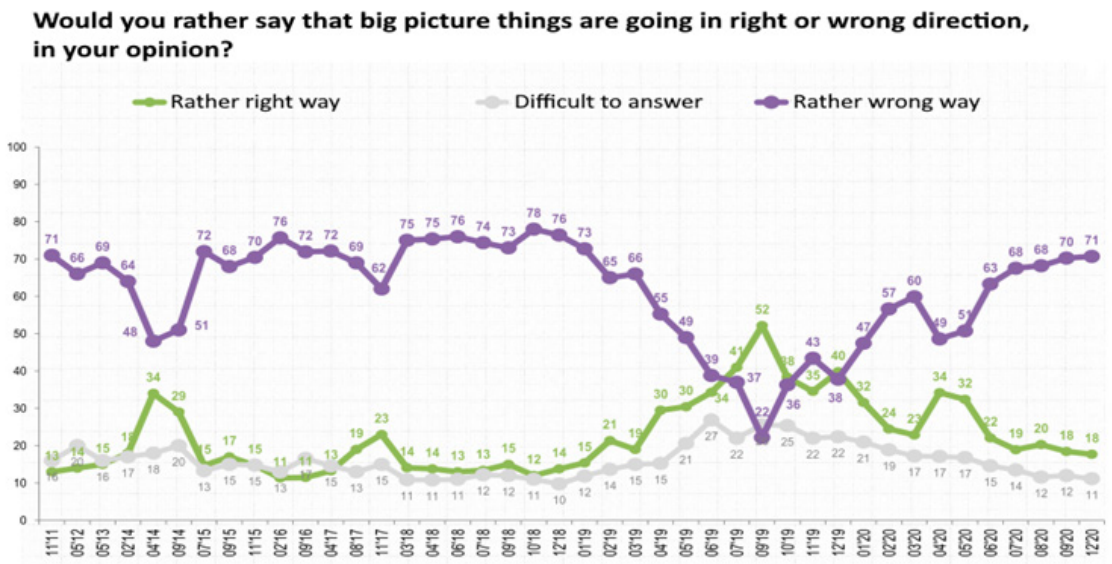

Figura 1. Satisfacción de las necesidades de los ciudadanos ucranianos por las autoridades y confianza en las decisiones del gobierno. Fuente: Elecciones locales (2020a).

En la encuesta de opinión pública realizada los días 16 y 20 de diciembre de 2020, en la se hizo la pregunta: ḋas cosas en Ucrania van en la dirección 
correcta o incorrecta?, más del $71 \%$ de los encuestados eligieron la segunda opción (Figura 1), en respuesta a la pregunta: ¿Cómo ha cambiado la situación económica en Ucrania durante el último medio año?, preguntada por los expertos a mediados de diciembre, el $74 \%$ delos participantes dijeron: "ha empeorado" (Actitudes sociales y políticas de la población, 2020). Por lo tanto, es posible establecer un patrón: la decepción está relacionada principalmente con los problemas sociales y económicos. La demanda de una "persona político-económica", un "hombre del alma", no es sólo un fenómeno cultural e histórico, sino también socioeconómico, que encarna la decepción del votante en las reformas, en el régimen político y en el sistema económico de nuevo.

Todo indica que la tendencia, durante las elecciones, revela que confianza en la dirección correcta en la que Ucrania se está moviendo está creciendo, por lo que, en abril 2014, durante las elecciones presidenciales, el 34\% de la población creía que el país se está moviendo en la dirección correcta, mientras que, en julio de 2015, el 71\% de los encuestados eligió la respuesta opuesta. Del mismo modo, durante las elecciones presidenciales de 2019, el 30\% de los encuestados dijo que Ucrania se estaba moviendo en la dirección correcta. Por otra parte, en septiembre del mismo año, más de la mitad de los encuestados (el 52\%) respondió positivamente (Figura 1) (Actitudes sociales y políticas de la población, 2021).

Pudieran pensarse que el votante, durante la campaña, se identifica con el candidato de su gusto, idealizándolo. El electorado ucraniano, busca desesperadamente el ideal de una "persona económica", en los líderes políticos ucranianos modernos. Ser de confianza y perderlo de nuevo, repitiendo el mismo camino. 
What is one reason why an economic crisis might occur in Ukraine?

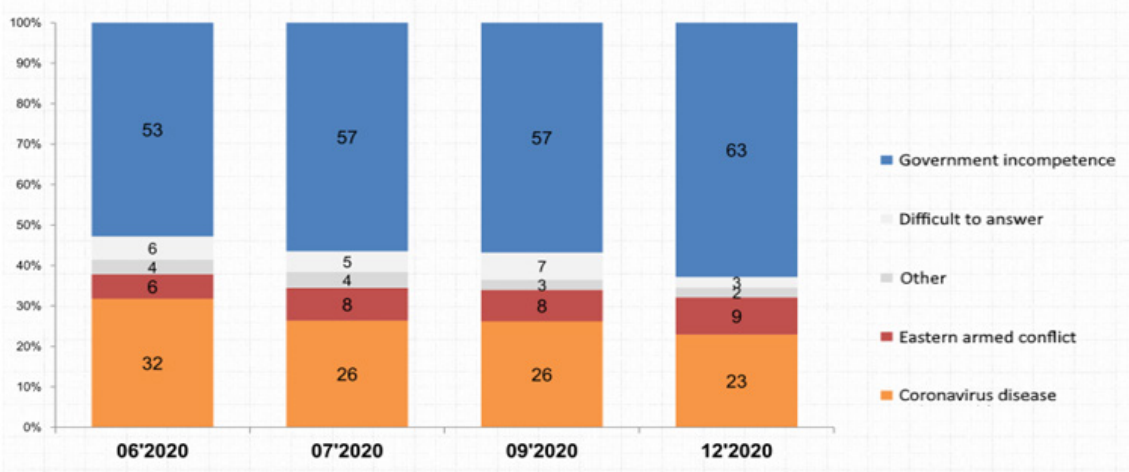

Figura 2. Resultados de la encuesta sobre la posible causa de la crisis económica en Ucrania. elaboración propia con base a la información proporcionada por: (Actitudes sociales y políticas de la población, 2020a)

Según la encuesta realizada a finales de 2020, cuando se le preguntó al elector "¿Cuál son las razones por las que podría haber una crisis económica en Ucrania?”, más del 63\% de los encuestados respondió que la incompetencia del gobierno podría ser la razón principal de una posible crisis. Hace de un año, el 53\% de los encuestados sostenía esta opinión (Actitudes sociales y políticas de la población, 2020b). (Figura 2)

El crecimiento del 10\%, en el nivel de desconfianza hacia el gobierno, en menos de medio año, revela una de las principales características de la autoconciencia, la percepción del mundo y la autoidentificación del votante ucraniano moderno, cuya esencia apunta a la necesidad de un enfoque fuerte y de gestión. En caso de que el votante se sienta decepcionado con la estrategia elegida por el gobierno, el nivel de confianza cae.

Cada vez que la esperanza en la persona de un votante ucraniano se vuelve más tenue, terminada desvaneciéndose con cada nueva decepción política, lo que puede configurar un sentimiento generalizado de descontento endémico y antipolítica.

El deseo de cambio es un marcador de la política contemporánea, la edad y la morfología geográfica. De una región a otra y de generación en generación, el "deseo de cambio" tiene un carácter completamente diferente. En el caso de la mayoría de los votantes occidentales y la generación más joven, se trata de un cambio cardinal hacia el lado progresista. Para 
los votantes orientales- hacia el lado regresivo; la cara del pasado está firmemente fijada en la conciencia de los votantes orientales. Sin embargo, la imagen de la "persona económica" sigue siendo una característica común de los votantes tanto de Occidente como de Oriente.

\section{El abstencionismo como característica de la política ucraniana moderna}

El abstencionismo, esto es, el comportamiento político caracterizado por la inacción, es decir, la evasión de cualquier participación política se ha convertido en una característica distintiva del sistema electoral ucraniano, que atestigua la desconfianza del votante ante la institución electoral (Shinkarenko, 2013). En la autoconciencia del votante moderno hay una idea: las elecciones no cambian nada, se desacreditan como herramienta de expresión de la voluntad individual y colectiva.

De una elección a otra, la participación electoral es cada vez menor. Esto se puede ver en la baja participación récord en las elecciones locales de 2020 , que fue de alrededor del $37 \%$. La participación electoral en las regiones occidentales de Ucrania fue mayor que en las regiones del sureste. Por ejemplo, si en la región de Ternopil y Lviv la participación fue de alrededor del 44\%, en las regiones sudorientales sólo alrededor de un tercio de los votantes acudieron a las elecciones. Como resultado, la participación de las regiones occidentales en el número total de votos aumentó del $27 \%$ al 31\% (Monitoreo de las elecciones locales, 2020, totales) (Figura 3).

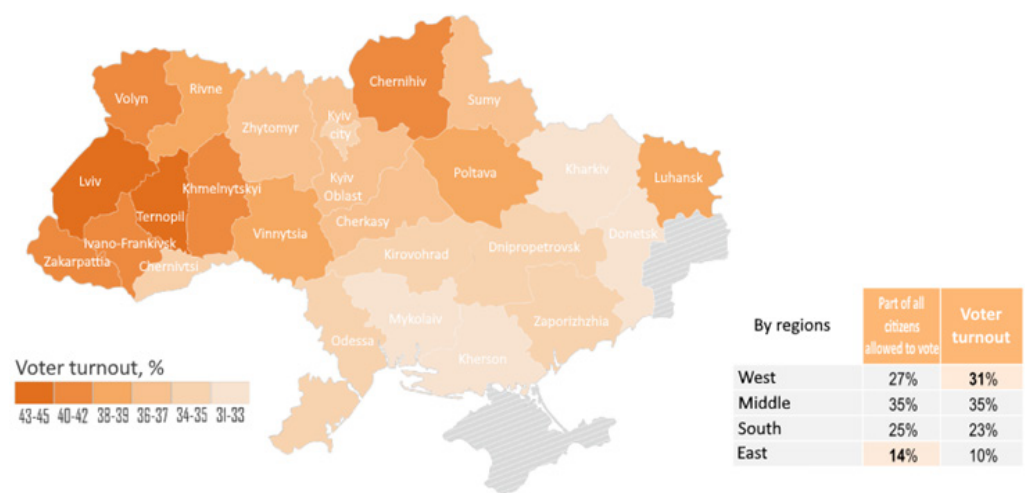


La aceptación hostil de las élites y de la política en general, manifestada en la alta proporción de papeletas deliberadamente estropeadas, de hecho, es una forma de protesta. En las elecciones presidenciales de 2019, el porcentaje de papeletas nulas en la segunda vuelta fue del $2 \%$. Observamos ahí un patrón claro: en el Este, la proporción de votos nulos es mucho menor, con un promedio de 1,5\%, en comparación con el 3\% en las regiones occidentales (Figura 4).

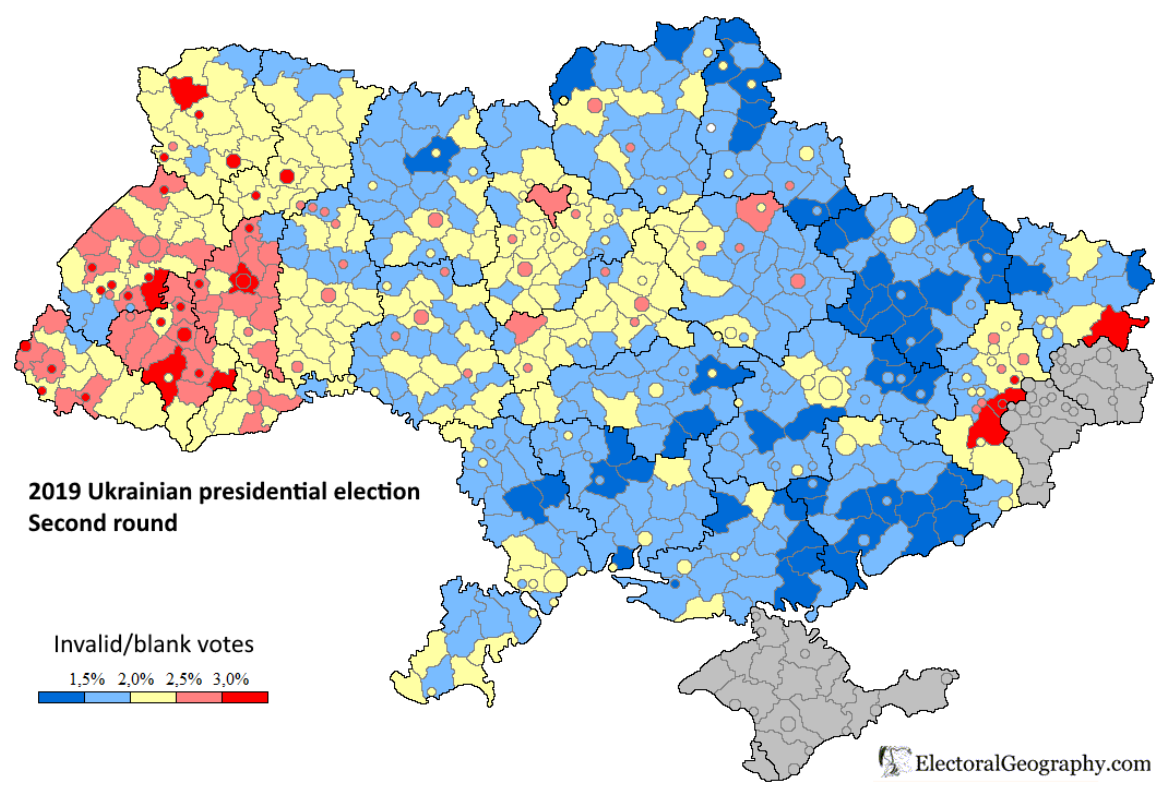

Figura 4 - Porcentaje de votos nulos o en blanco en la segunda vuelta de las elecciones presidenciales de Ucrania de 2019. Fuente: Geografía electoral 2.0. Ucrania. Elecciones presidenciales (2019).

Además, en las zonas urbanas, el número de papeletas nulas es significativamente mayor que en las zonas rurales. Anteriormente, el sistema electoral brindaba oportunidades para la protesta pasiva a través de la cláusula "contra todos", pero esta ha sido eliminada. Esto se correlaciona con la participación, donde es posible trazar una tendencia - las zonas occidentales, en promedio, tienen una mayor participación, al igual que las zonas urbanas tienen una mayor participación que las zonas rurales (Elecciones locales, 2020b). Todo esto da fe del mayor potencial de protesta de Occidente y de la relativa pasividad de las regiones oriental 
y sudoriental. Todo esto refleja diferencias electorales-geográficas, sin embargo, en ambos casos, se convierte en una prueba de la decepción general en el sistema político, solo que los métodos y formas de protesta difieren, la protesta pasiva en el Este y la protesta activa en el Oeste.

\section{El fenómeno del votante moderno en el contexto de la estructura electoral de la edad}

La estructura de los votos dependiendo de la edad se remonta a los jóvenes políticos del electorado bajo la hipótesis de que no están suficientemente formados "edad política". La inmadurez de la comprensión del enfoque político estatal se observa tanto por parte de los votantes como de los elegidos. Esta naturaleza apolítica está relacionada con el hecho de que, en el contexto regional, también es posible rastrear una tendencia: de Oeste a Este, la proporción del electorado de más edad en el número total de votos está aumentando, allí la proporción de votantes mayores de 50 años era del $60 \%$, con diferencias insignificantes en la estructura de edad de la población.

Con el envejecimiento de la nación y la crisis demográfica, no es sorprendente que la actividad electoral de la población mayor de 50 años represente la mayor parte del voto. Sin embargo, con la proporción de la población mayor de 50 años en el número total del electorado en el $45 \%$, la desproporción se hace evidente (Elecciones locales, 2020, análisis de la estructura por edades de los votantes) (Figura 5).

\section{Share of the Voter Population, by Age}

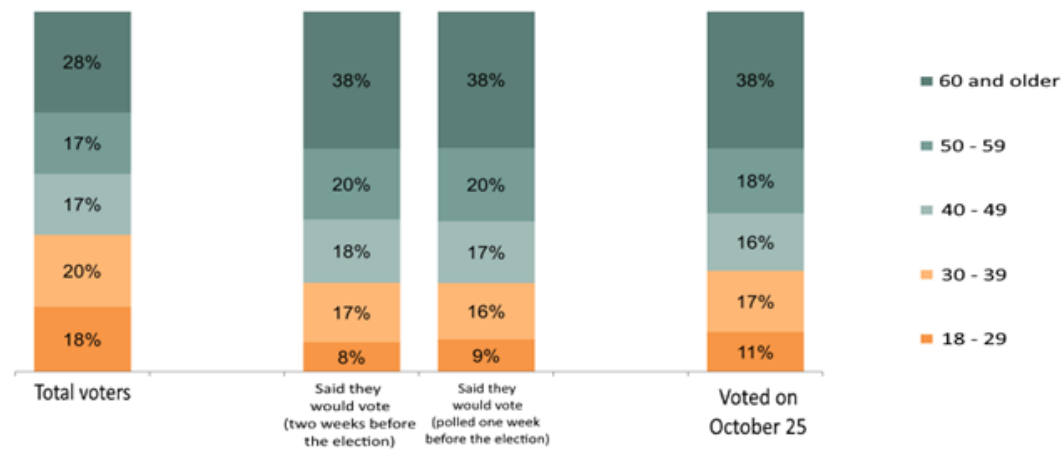

Figura 5 - Proporción de la población votante por edad. Fuente: (Elecciones locales, 2020b). 
En los distritos rurales, la abrumadora mayoría de los candidatos son locales, en una comunidad pequeña, de clase media, bien establecidos en posiciones gubernamentales o económicas. La imagen del "muchacho de la granja" es claramente evidente en el abrumador número de candidatos ganadores en los distritos rurales. La peculiaridad es que, en las zonas rurales, la desproporción hacia el electorado de más edad es entre un $3 \%$ y un $4 \%$ menor, siempre que, por término medio, la estructura demográfica de la población sea mayor.

\section{Share of the Voter Population, by Age and Type of Settlement}

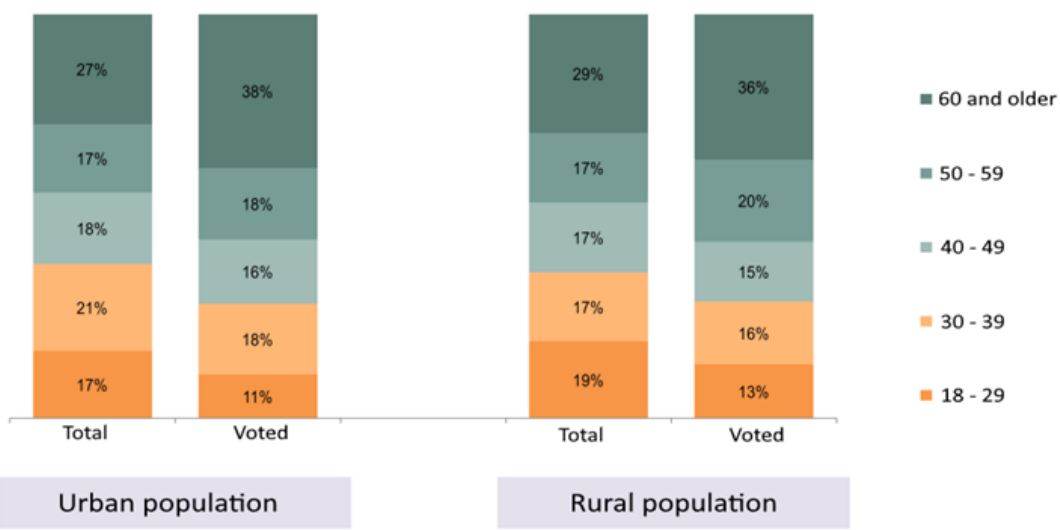

Figura 6 - Proporción de la población votante por edad y tipo de asentamiento. Fuente: (Elecciones locales, 2020b).

El electorado, de entre 18 y 29 años, fue el más pasivo en las últimas elecciones locales. En una proporción, en la estructura general de edad de los electores, $18 \%$, una proporción del electorado de 18-29 años, en la estructura general de votos significa sólo el $11 \%$ (Seguimiento de las elecciones locales, 2020, totales). Para la generación joven - el empleo el día de las elecciones y no saber por quién votar, se convirtió en las principales razones de la ignorancia del proceso electoral (Seguimiento de las Elecciones Locales, 2020, totales). (Figura 6). También podemos suponer que una de las razones por las que los jóvenes ignoran las elecciones es su deseo de migrar.

La socialización política como proceso de inclusión de un individuo en la sociedad se produce a través de la asimilación de la experiencia de 
generaciones propias y de otras generaciones fijadas en la cultura de la sociedad. Para los jóvenes y parte del grupo de mediana edad, la socialización política tuvo lugar en el tiempo postsoviético en el cual se da la formación de Ucrania como un estado independiente (Andrushchenko, 2015), situación que, en gran medida, influyó en la perspectiva, las características sociales y psicológicas de la llamada "generación de la libertad". La destrucción o degradación de las antiguas instituciones públicas tradicionales, el libre acceso a la información marca una huella en el proceso de socialización política del electorado del grupo de edad de 18 a 29 años.

La imagen del político para la generación más joven, formada en las realidades de la era de Internet, es diferente, a las generaciones mayores, lo que da paso a un político directamente cercano al electorado. Para las generaciones anteriores, la imagen de un "hombre de alma", era una condición necesaria para la percepción de un candidato, mientras que, para la generación más joven, la imagen de las generaciones pasadas ha sido reemplazada por la imagen de un "hombre de enfoque", un hombre dispuesto a defender ideas de avanzada, abierto y comprensible para el electorado.

La visión del mundo de las generaciones más jóvenes y mayores parecía tener diferencias significativas. Sin embargo, de hecho, la imagen del "hombre del alma" sólo ha recibido cambios menores. El cardiocentrismo, en la cosmovisión de la "generación de la libertad", ha conservado su forma anterior, cambiando al mismo tiempo algunas características: la simplicidad del alma ha cambiado a la apertura; principios morales idealizados, integridad y honestidad, han sido reemplazados por la ideología; sabiduría, por la capacidad de ajustarse a las tendencias. Con diferencias aparentemente visibles, el cardiocentrismo ucraniano se ha convertido en la base de la cosmovisión de la generación ucraniana moderna también. La actitud hacia el papel del estado, por parte de todas las generaciones se mantuvo sin cambios, el votante ve su papel en la esfera social y económica, delegando funciones significativas en el estado, en tanto eje central del sistema político y del orden social.

Cambios de edad, en la percepción del "candidato ideal" por generaciones, causados por el hecho de que el individualismo, en mayor medida, es típico para el electorado joven en la edad de 18-29 años, mientras que, para los mayores, por el contrario, los valores colectivistas no son más relevantes (Shaygorodsky, 2020).

Las redes sociales, como forma de obtener información para la generación más joven, han sustituido a los medios de comunicación tradicionales, que siguen siendo relevantes para los grupos de edad de 30 años o más. La naturaleza de la percepción de la información es el factor más importante de la socialización política, forma valores individuales o colectivos. Evidencia de diferencias morfológicas en la percepción, así como en ausencia de 
diferencias fundamentales, puede ser la estructura electoral-geográfica de la población, donde hay una clara tendencia: la disparidad de edad, en la estructura de las personas que votaron disminuye, de oeste a este.

En las regiones orientales, los partidos populares en su mayoría de izquierda moderada, en virtud de su ideología colectivista, local y nacional, que explotan la imagen de "persona económica", en Occidente, en su mayoría populares son partidos de derecha radical o moderada, de naturaleza individualista, que en su mayor parte explotan la visión de la generación más joven. Para el electorado de 18 a 29 años, el futuro de Ucrania está en avanzar, en las reformas de acuerdo con el modelo occidental y, para los mayores, en volver al viejo modelo de desarrollo. Sin embargo, en ambos casos, hay decepción por parte de la generación más joven en el bajo ritmo de aplicación y el débil efecto socioeconómico de las reformas políticas y económicas y; por parte de la generación más antigua, en el camino elegido (Guseva, 2020).

La generación de la mediana edad (30-49 años) es un vínculo de transición, que maduró en el cambio de una era, en consecuencia, los representantes del grupo de edad electoral media se convirtieron en algo entre los jóvenes y la generación mayor, encarnando la visión del mundo de ambas generaciones. Toman una posición centrista en el conflicto de la cosmovisión, van a elecciones, a diferencia de la generación más joven, pero no son tan activos como la generación mayor. Con un nivel medio de apoliticismo, más cercano a la imagen de un "Hombre del alma" que a la de un "hombre más cercano".

\section{El ascenso de partidos regionales en el contexto del fenómeno moderno del votante}

La búsqueda de una "persona económica" se ha convertido en el principio de los votantes, como lo demuestra el surgimiento de los partidos regionales, encarnados no a partir de una ideología política tradicional, sino en la figura de un alcalde bien establecido, a imagen de un líder fuerte, un luchador honesto y de principios contra las élites centrales que representa los intereses locales de la gente común. La llamada "feudalización" o "latifundismo" se manifiesta en muchos factores. Sin embargo, quizás la más importante es la división del campo electoral en partidos regionales y nacionales.

Los partidos nacionales representan la ideología del Estado, instituyendo el vector para el establecimiento del Estado ucraniano. Los partidos regionales, a los ojos del votante moderno, encarnan la imagen de una "persona económica". Según los resultados de las elecciones locales de 2020, los partidos locales recibieron más del 20\% del número total 
de votos, y la calificación general de los cinco partidos parlamentarios nacionales disminuyó del $78 \%$ al $51 \%$ (Seguimiento de las elecciones locales, 2020, totales). Bloque Kernes - Éxito Járkov con un resultado del 60\%, Confiar en los hechos, el alcalde de Odessa Gennady Trukhanov con el 54\%, Vadim Boychenko partido- alcalde de Mariupol con el 64\%, Partido de Vladimir Buryak "Unidad", que ganó en la primera ronda con el resultado del 60\% (Información sobre los cargos de jefes de ciudad, municipio y aldea, 2020) - un desfile de victorias de los partidos locales nombrados, en las elecciones de los jefes de las ciudades, especialmente en las ciudades grandes y medianas, es una demostración de la decepción total del electorado en los partidos nacionales. Sin embargo, tal división, caracteriza sólo los problemas nacionales, "puntos dolorosos" de la política ucraniana, sin embargo, también demuestra que la imagen de "persona económica", fiablemente fijado en las simpatías electorales.

En la abrumadora mayoría de los votantes ucranianos, no importa en absoluto la ideología del partido, la personalidad del candidato es lo realmente importante. El político que en condiciones de formación del sistema de partidos-democrático es el que más encaja en la imagen idealizada por los votantes y obtiene el mayor apoyo (Goncharenko, 2020). Esto es sobre lo que se basa la campaña electoral moderna, la ideología es de importancia secundaria, es sólo una cubierta en la que la imagen de un líder: honesto, responsable, amable y simple "un hombre del alma" está envuelto.

\section{Conclusiones}

Así, en la autoconciencia del votante moderno, la política, se personifica con la kakistocracia --el poder de los peores representantes de la sociedad--, los menos calificados, los más inescrupulosos, deshonestos y no sabios. Este estado de cosas afecta negativamente el proceso de formación del sistema electoral en Ucrania, donde hay una responsabilidad tanto del que elige como del que es elegido. Podemos suponer que el votante ucraniano, en primer lugar, es una persona insegura sobre el futuro, no confía en el sistema político, en los actores políticos ni en el Estado, lo que se personifica, la decepción es la característica misma del fenómeno del votante moderno.

Es la imagen de una "persona económica" que es ante todo la imagen de un estadista, cuya actividad se basa en principios morales firmes, un líder sabio, cuya característica principal es la integridad y la honestidad.

Se descubrió que la formación de la personalidad del elector pasó en tres etapas, desde los primeros pasos de la formación de la experiencia electoral en el imperio zarista a través de su colapso en el período soviético y la etapa moderna de la vida del elector. El electorado se estudiaba en función de la edad y el lugar de residencia. 
El fenómeno del votante moderno radica en las realidades históricohistóricas y sociopolíticas que han moldeado la cosmovisión electoral, dentro de un solo individuo. En su esencia, la "persona económica" es una continuación evolutiva del "hombre del alma". El cardiocentrismo, en absoluto, no se convirtió en una cosa del pasado durante la época soviética, sino que se transformó más bien en una parte de la teoría político-filosófica y cultural general, marcando los rasgos característicos del fenómeno del elector moderno.

La estructura electoral de los votantes refleja las diferencias geográficas y de edad, sin embargo, en ambos casos hay decepción en el sistema político, y como resultado hay una protesta social constante. Además, se establece que el ascenso de los partidos regionales en las últimas elecciones se produjo a expensas de la imagen artificialmente formada de "persona económica", que entiende y siente el electorado en el campo.

\section{Referencias Bibliográficas}

ACTITUDES SOCIALES Y POLÍTICAS DE LA POBLACIÓN (16-20 de diciembre). 2020a. Disponible en línea. En: http://ratinggroup.ua/ $\mathrm{ru} /$ research/ukraine/obschestvenno-politicheskie_nastroeniya_ naseleniya_16-20_dekabrya.html. Fecha de consulta: 12/03/2021. (En ucraniano)

ANDRUSHCHENKO, Tetiana. 2015. "Potencial adaptativo de la socialización política" En: Revista del Instituto para el Desarrollo Infantil. Ser: Filosofía, Pedagogía, Psicología. No. 37, pp. 105-109. (En ucraniano)

ELECCIONES LOCALES. 2020a. Disponible en línea. En: https://www.cvk. gov.ua/pls/vm2020/wmoo1ptoo1fo1 $=695 \cdot \mathrm{html}$. Fecha de consulta: 12/03/2021. (En ucraniano)

ELECCIONES LOCALES. 2020b. Análisis de la estructura por edades de los votantes. Disponible en línea. En: http://ratinggroup.ua/research/ ukraine/mestnye_vybory_2020_analiz_vozrastnoy_struktury_ izbirateley.html. Fecha de consulta: 24/01/2021. (En ucraniano)

FIDROWSKA, María. 2019. "Cardiocentrismo de la filosofía ucraniana" En; Espiritualidad como componente de estado ucraniano: una colección de trabajos de investigación por los materiales de la conferencia científicopráctica internacional, pp. 201-202. Járkov, Ucrania. (En ucraniano)

GONCHARENKO, Natalia. 2020. "Sistema de partidos de Ucrania: etapas de formación, perspectivas de desarrollo" En: Dimensiones socioeconómicas, políticas y humanitarias del desarrollo nacional y local. No. 2, pp. 159-165. (En ucraniano) 
GUSEVA, Natalia. 2020. "Irracionalidad electoral” En: Problemas reales de la psicología. Vol.1, No. 56, pp. 67-71. (En ucraniano)

INFORMACIÓN SOBRE LOS CARGOS DE JEFES DE CIUDAD, MUNICIPIO Y ALDEA. 2020. Disponible en línea. En: https://www.cvk.gov.ua/pls/ vm2020/pvmoo3ptoo1fo1=695ptoo_too1fo1=695.html. Fecha de consulta: 12/03/2021. (En ucraniano)

SABADUKHA, Volodymyr. 2012. "Idea nacional ucraniana y concepto de ser personal” En: Wanderer. No. 06, pp. 4-14. (En ucraniano)

SAMCHUK, Zoreslav. 2017. "Formation of a civilizational paradigm for the development of Ukraine: Theoretical and Methodological Analysis of Prospects and Restrictions" In: Scientific Notes of the Kuras Institute of Political and Ethnic Studies of the National Academy of Sciences of Ukraine. No. 04, pp. 53-70.

SEGUIMIENTO DE ELECCIONES LOCALES 2020: TOTALES. 2020. Disponible en línea. En: http://ratinggroup.ua/research/ukraine/ monitoring_mestnyh_vyborov_2020_itogi.html. Fecha de consulta: 12/12/2020. (En ucraniano)

SHAYGORODSKY, Yurii. 2020. "Factores mediáticos-psicológicos de elección electoral 2019" En: Revista Científica de la Universidad Pedagógica Nacional Dragomanov. Vol. 02, No. 28, pp. 30-42. (En ucraniano)

SHINKARENKO, Olena. 2013. "Marketing y ausentismo electorales" En: Facetas. No. 10, pp. 121-126. (En ucraniano)

SOBRE EL “CARDIOCENTRISMO” DEL VOTANTE UCRANIANO. 2020. Disponible en línea. En: https://m.day.kyiv.ua/uk/article/tema-dnyapodrobyci/pro-kardiocentryzm-ukrayinskogo-vyborcya. Fecha de consulta: 23/o1/2021. (En ucraniano)

ACTITUDES SOCIALES Y POLÍTICAS DE LA POBLACIÓN (2-4 de enero). 2020b. Disponible en línea. En: http://ratinggroup.ua/ru/research/ ukraine/obschestvenno-politicheskie_nastroeniya_naseleniya_2-4_ yanvarya.html. Fecha de consulta: 24/01/2021. (En ucraniano)

GEOGRAFÍA ELECTORAL 2.o. UCRANIA. ELECCIONES PRESIDENCIALES 2019. Disponible en línea. En: https://www.electoralgeography.com/ new/ru/ukraine2019/2019-ukraine-second-invalid-raions.png. Fecha de consulta: 15/02/2021. (En ucraniano) 
Vol. 39 N $^{\circ} 71$

Esta revista fue editada en formato digital y publicada en diciembre de 2021, por el Fondo Editorial Serbiluz, Universidad del Zulia. Maracaibo-Venezuela 